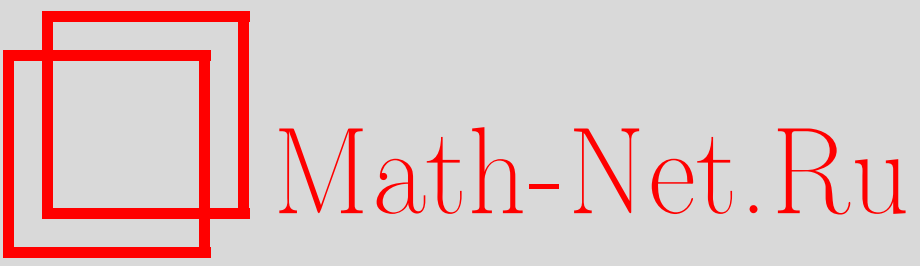

Н. А. Свешников, Э. Г. Тимошенко, Граничные условия и механизм конфайнмента в калибровочной теории поля при конечной температуре, ТМФ, 1998, том 117, номер 2, 206-220

DOI: https://doi.org/10.4213/tmf927

Использование Общероссийского математического портала Math-Net.Ru подразумевает, что вы прочитали и согласны с пользовательским соглашением

http://www. mathnet.ru/rus/agreement

Параметры загрузки:

IP: 52.23 .180 .231

26 апреля 2023 г., 18:22:26 
ТЕОРЕТИЧЕСКАЯ

И МАТЕМАТИЧЕСКАЯ

ФИЗИКА

Том 117, № 2

ноябрь, 1998

(C) 1998 г. Н.А. Свешников, Э.Г. Тимошенко*

\section{ГРАНИЧНЫЕ УСЛОВИЯ И МЕХАНИЗМ КОНФАЙНМЕНТА В КАЛИБРОВОЧНОЙ ТЕОРИИ ПОЛЯ ПРИ КОНЕЧНОЙ ТЕМПЕРАТУРЕ}

Построена гамильтонова формулировка неабелевой калибровочной теории в конечной области в обобщенной 3 -мерной калибровке Фока-Швингера с учетом поверхностных членов. Изучена зависимость статистической суммы теории от граничного значения продольной компоненты электрического поля, которая в силу закона Гаусса в данной калибровке совпадает с потоком электрического поля через бесконечно малый элемент границы. Установлена связь такой зависимости с механизмом фазового перехода конфайнмент-деконфайнмент. В фазе конфайнмента поток хромоэлектрического поля через произвольный элемент границы равен нулю, что является условием синглетности относительно группы остаточных калибровочных преобразований и физически означает невозможность наблюдения цветных объектов на пространственной бесконечности. Анализ неабелевой теории предваряется рассмотрением более простой задачи о квантовой электродинамике с внешней плотностью заряда в сферической области, где эффективная статистическая сумма вычисляется точно.

$\mathrm{C}$ чувством глубокой скорби и невозвратимой потери мне пришлось завершить написание этой работы в одиночку. В лище Никиты Алексеевича Свешникова теоретическая физика потеряла чрезвычайно талантливого ученого, прекрасного педагога и замечательного человека. Несмотря на значительность влияния идей Никиты Алексеевича на многих его коллег и студентов, одним из которых я был, глубина этих идей, возможно, будет до конца оценена лишь последующими поколениями.

По-видимому, эту статью можно рассматривать как логический итог нашего совместного сотрудничества по изучению проблемы конфайнмента. И хотя, с моей точки зрения, она проливает новый свет на одну из самых сложных проблем в физике элементарных частиц, это лишь небольшой шаг вперед. Н. А. Свешников обладал замечательным даром видения скрытой математической красоты физической реальности, красоты, которая выражает сущность мира.

Э.Г. Тимошенко

\section{1. ВВЕДЕНИЕ}

Проблема конфайнмента в неабелевой калибровочной теории уже несколько десятков лет привлекает самый живой интерес исследователей и является одной из самых

* Department of Chemistry, University College Dublin, Ireland. E-mail: Edward.Timoshenko@ucd.ie 
актуальных для обоснования стандартной модели сильных и электрослабых взаимодействий. Несмотря на значительный прогресс в изучении многих важных аспектов конфайнмента $[1,2]$, в научном сообшестве до сих пор не сушествует согласия между различными подходами, которые пытаются вывести конфайнмент из первых принципов квантовой хромодинамики (КХД).

Наличие конфайнмента означает присутствие в системе дальнодействия, которое является источником линейно растушего потенциала. Причина подобного явления в локальной теории в принципе понятна. Действительно, благодаря калибровочной инвариантности в системе имеется связь, представляющая собой закон Гаусса. При выборе физической калибровки связь можно разрешить и выразить компоненту электрического поля, нормальную к границе $E_{\|}$, в виде некоторого интегрального оператора. Подстановка последнего выражения в гамильтониан системы приводит к эффективному дальнодействию и нелокальности уравнений движения физических полей.

С другой стороны, нелокальность - это источник граничной нетривиальности теории. Действительно, граничное значение переменной $E_{\|}$является не чем иным, как потоком цветового заряда через границу в рассматриваемом направлении. Граничное условие на $E_{\|}$приобретает статус связи, т.к. требует обращения в нуль определенной интегральной конструкции от физических переменных. Иными словами, поверхностные члены превращаются в объемные. Поэтому возникает необходимость в развитии последовательной схемы учета поверхностных членов.

Присутствие нефизических степеней свободы в ковариантной формулировке теории Янга-Миллса заметно усложняет анализ проблемы граничных условий благодаря вносимому ими дополнительному произволу. Представляется разумным работать в некоторой физической калибровке, обеспечивая тем самым положительную определенность гильбертова пространства состояний. Обобщенная калибровка Фока-Швингера (ФШ) выделена для анализа нашей проблемы благодаря ее следующим замечательным свойствам: гауссова связь может быть в ней разрешена в явном виде; гамильтониан системы оказывается полиномом четвертой степени относительно канонических переменных; только продольные компоненты хромоэлектрического и магнитного полей содержат неабелевы и нелокальные конструкции; приближение среднего поля является нетривиальным для коллективных переменных из-за квазиклассического характера фоновых полей напряженностей.

Известно, что вследствие безмассовости глюонов теория Янга-Миллса содержит серьезные инфракрасные расходимости. Для аккуратного обращения с ними на первом этапе необходимо ввести инфракрасную регуляризацию, которая удаляется в окончательном результате. Наиболее простой и физически наглядной является регуляризация системы с помощью заключения ее в конечную область. Заметим, что такая процедура всегда необходима для построения теории при конечной температуре, т.к. из-за трансляционной инвариантности логарифм статистической суммы пропорционален объему пространства. Поэтому обычно систему заключают в конечную область $V$ и накладывают некоторые условия на все поля на границе области $\partial V$. Затем, чтобы проанализировать зависимость от граничных условий, естественно рассмотреть функционал

$$
Z[\chi]=\operatorname{Tr}\left(e^{-\beta H_{V}} \delta\left(\left.\phi\right|_{\partial V}-\chi\right)\right),
$$

где $H_{V}$ - гамильтониан системы в объеме $V, \beta$ - обратная температура, $\phi$ - набор ка- 
нонических переменных и функции $\chi$, определенные на границе $\partial V$, задают граничные условия Дирихле для них. В этом контексте мы будем называть $Z[\chi]$ әффективной статистической суммой по аналогии с введением понятия эффективного действия.

Стандартным предположением в теории поля является отсутствие поверхностных членов, возникающих при интегрировании по частям. Для задач в конечной области это обеспечивается надлежащим выбором граничных условий, которым удовлетворяют поля на поверхности. В пределе бесконечного объема достаточно предполагать, что поля убывают достаточно быстро на пространственной бесконечности. Такие требования, безусловно, являются вполне оправданными для многих ситуаций в теории поля [3].

Теория с калибровочной симметрией, как мы отметили выше, обладает рядом специфических свойств. Поэтому стандартные предположения о поведении полей на бесконечности, строго говоря, не очевидны. Таким образом, возникает необходимость получать уравнения движения с учетом поверхностных членов, но, кроме того, значения полей на границе должны фигурировать как равноправные гамильтоновы переменные. Более того, добавка поверхностного члена к гамильтониану, необходимая для учета поверхностной связи, приобретает значимый физический смысл.

Задача учета границы в гамильтоновом формализме естественно возникает, например, при рассмотрении поверхностных волн в гидродинамике [4-6], где поверхностные члены имеют непосредственный физический смысл. В работе Редже и Тейтельбойма [7] было показано, что поверхностные члены могут играть существенную роль в теории гравитации, где этот вопрос до сих пор является предметом активного изучения $[8,9]$.

В последнее время интерес к граничным эффектам в различных теориях поля заметно возрос [10, 11]. В рамках алгебраической квантовой теории поля [12] было показано, что некоторые 2-мерные модели обладают нетривиальной динамикой переменных на бесконечности, что является источником нетривиального физического эффекта динамической генерации массы.

В температурной теории естественно ожидать, что $Z[\chi]$ и, следовательно, любые термодинамические функции становятся не зависимыми от конкретного выбора граничных условий в термодинамическом пределе $V \rightarrow \infty$. Однако опять же можно усомниться, что подобное поведение сохранится для систем с дальнодействующими взаимодействиями. Изучение данной зависимости от существенных граничных условий в калибровочной теории поля и является основным предметом этой работы.

Зная лишь зависимость эффективной статистической суммы от граничного условия на $E_{\|}$в термодинамическом пределе, мы можем сформулировать простой критерий конфайнмента: $Z[\chi] \propto \prod_{\widehat{\mathbf{x}}} \delta(\chi(\widehat{\mathbf{x}}))$. Последнее условие означает, что цветовой поток равен нулю в любом направлении на пространственной бесконечности для любого состояния, принадлежащего пространству состояний системы в фазе конфайнмента.

\section{2. ОБОБШЕННАЯ КАЛИБРОВКА ФОКА-ШВИНГЕРА}

Пусть $V_{R}$ - регулярная область в $\mathbf{R}^{3}$, топологически эквивалентная шару, с гладкой границей $\partial V_{R}$. Удобно выбрать такую криволинейную систему координат $\mathbf{X}$ в области $V_{R}$, чтобы на поверхностях $\partial V_{R}$ первая координата была постоянна и равна параметру $R$, который будет играть роль инфракрасного обрезания в нашем рассмотрении, т.е. $\partial V_{R}=\left\{\mathbf{X}: X_{1}(\mathbf{x})=R=\mathrm{const}\right\}$, где координаты в декартовой системе обозначаются 
строчными буквами х. Поле нормалей к границе, взятое в совокупности при всех значениях $R$, образует гладкое векторное поле во всей области.

Локальный ортонормированный криволинейный базис может быть представлен в виде

$$
e_{i}^{(k)}=\frac{1}{h_{k}} \frac{\partial x_{i}}{\partial X_{k}}, \quad h_{k}=\left(\sum_{i=1}^{3}\left(\frac{\partial x_{i}}{\partial X_{k}}\right)^{2}\right)^{1 / 2}, \quad h \equiv \prod_{i=1}^{3} h_{i} .
$$

Компоненты векторов в криволинейном базисе для отличия их от компонент в декартовом базисе обозначаются буквами, заключенными в скобки, $A_{(k)}=e_{(k)}^{i} A_{i}$ и $\partial_{(k)} \equiv$ $\left(1 / h_{k}\right)\left(\partial / \partial X_{k}\right)$. Вектор $\mathbf{e}_{(1)}$, очевидно, и задает описанное нами поле нормалей. Естественно ввести $(2+1)$ разложений компонент векторов на продольную и поперечные составляюшие (обозначаемые греческими буквами): $i \rightarrow(1, \alpha), \alpha=2,3$. В качестве векторной записи для "греческих" компонент криволинейных координат мы будем использовать обозначение $\mathbf{X}=\left(X_{2}, X_{3}\right)$.

Калибровочная теория в конечном области $V_{R}$ принимает наиболее простой вид в специальной калибровке, вид которой согласован с формой границы. А именно, потребуем, чтобы компонента калибровочного поля в направлении поля нормалей равнялась нулю в каждой точке:

$$
\mathbf{e}_{(1)}(\mathbf{x}) \mathbf{A}(t, \mathbf{x})=0, \quad \mathbf{A}=\mathbf{A}_{\perp}, \quad \mathbf{A}_{\perp}=P \mathbf{A}, \quad P=\mathbf{1}-\mathbf{e}_{(1)} \otimes \mathbf{e}_{(1)} .
$$

Такая калибровка называется обобшенной калибровкой $ш$.

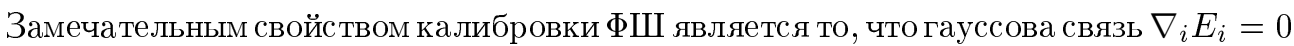
разрешается в ней в явном виде

$$
E_{(1)}=-\frac{h_{1}}{h} \int_{X_{1}^{(0)}}^{X_{1}} d X_{1}^{\prime}\left(h \Phi_{\perp}\right)\left(X_{1}^{\prime}, \breve{\mathbf{X}}\right),
$$

выражая продольную компоненту электрического поля через поперечную часть связи

$$
\Phi_{\perp} \equiv \nabla_{i} E_{\perp i}=\frac{h_{\alpha}}{h} \nabla_{(\alpha)}\left(\frac{h}{h_{\alpha}} E_{(\alpha)}\right) .
$$

Нижний предел интегрирования $X_{1}^{(0)}$ здесь равен некоторой константе, которую, как мы покажем ниже, целесообразно выбрать в точке, соответствующей началу системы координат.

Тождество $\nabla_{i} G_{i}=0, G_{i} \equiv \nabla_{j} F_{i j}$, можно разрешить, выразив $G_{(1)}$ через поперечные компоненты данного вектора,

$$
G_{(1)}=-\frac{h_{1}}{h} \int_{\bar{X}_{1}^{(0)}}^{X_{1}} d X_{1}^{\prime}\left(h \nabla_{i} G_{\perp i}\right)\left(X_{1}^{\prime}, \breve{\mathbf{X}}\right) .
$$

Далее, одна из компонент тождеств Бианки записывается в виде

$$
\nabla_{i} B_{i}=0, \quad B_{k}=\frac{1}{2} \epsilon_{i j k} F_{i j},
$$

что позволяет выразить продольную компоненту магнитного поля следующим образом:

$$
B_{(1)}=-\frac{h_{1}}{h} \int_{X_{1}^{(0)}}^{X_{1}} d X_{1}^{\prime}\left(h \nabla_{i} B_{\perp i}\right)\left(X_{1}^{\prime}, \breve{\mathbf{X}}\right) .
$$


Можно показать, что остальные две компоненты тождеств Бианки, $\epsilon_{i j k} e_{(\alpha)}^{i} \nabla_{j} E_{k}=0$, эквивалентны тождествам

$$
\frac{1}{h_{\alpha}} \frac{\partial}{\partial X_{1}}\left(h_{\alpha} E_{(\alpha)}\right)=\nabla_{(\alpha)}\left(h_{1} E_{(1)}\right) .
$$

В качестве простого примера рассмотрим сферические координаты $X_{1}=r, X_{2}=\phi$, $X_{3}=\theta$, в которых параметры Ламе имеют особенно простой вид,

$$
h_{1}=1, \quad h_{2}=X_{1} \sin X_{3}, \quad h_{3}=X_{1} .
$$

Важным свойством сферической (обычной) калибровки ФШ, которое заметно упрощает формулы, является то, что $\mathbf{e}_{(i)}$ не зависят от $X_{1}$. Вектор нормали при этом равен единичному радиус-вектору $\mathbf{e}_{(1)}=\widehat{\mathbf{x}}$.

\section{3. ГАМИЛЬТОНОВ ФОРМАЛИЗМ ДЛЯ КАЛИБРОВОЧНОЙ ТЕОРИИ В КОНЕЧНОЙ ОБЛАСТИ}

Для некоторого класса полевых моделей в работе [11] было предложено обобшение гамильтонова формализма, основная идея которого состоит в том, чтобы добавкой некоторых поверхностных членов модифицировать скобки Пуассона так, чтобы они строго, а не с точностью до поверхностных членов, как в традиционном формализме, удовлетворяли тождествам Якоби. Мы ограничимся формулировкой для случая, когда гамильтониан системы содержит производные канонических переменных не выше первого порядка. Рассмотрим класс локальных функционалов от канонических переменных вида

$$
F=\int_{V} d \mathbf{x} f\left[\varphi^{A} ; \varphi_{, i}^{A}\right] .
$$

Пусть канонические переменные обладают канонической скобкой Пуассона $\left\{\varphi^{A}(\mathbf{x})\right.$, $\left.\varphi^{B}\left(\mathbf{x}^{\prime}\right)\right\}=I^{A B} \delta\left(\mathbf{x}, \mathbf{x}^{\prime}\right)$, где $I^{A B}$ - стандартная симплектическая матрица со свойством $I^{2}=-1, I=$ const. Вариация такого функционала при изменении канонических переменных может быть записана в виде

$$
\delta F=\int_{V} d \mathbf{x}\left(\mathcal{E}_{A}(F) \delta \varphi^{A}+\partial_{i}\left(\mathcal{E}_{A}^{i}(F) \delta \varphi^{A}\right)\right),
$$

где эйлеровы производные нулевого и первого порядков равны

$$
\mathcal{E}_{A}(F)=\frac{\partial f}{\partial \varphi^{A}}-\partial_{i} \frac{\partial f}{\partial \varphi_{, i}^{A}}, \quad \mathcal{E}_{A}^{i}(F)=\frac{\partial f}{\partial \varphi_{, i}^{A}} .
$$

Определим скобку Пуассона двух произвольных функционалов $F$ и $H$ :

$$
\begin{aligned}
\{F, H\}= & \int_{V} d \mathbf{x}\left(\mathcal{E}_{A}(F) I^{A B} \mathcal{E}_{B}(H)+\right. \\
& +\partial_{i}\left(\mathcal{E}_{A}^{i}(F) I^{A B} \mathcal{E}_{B}(H)+\mathcal{E}_{A}(F) I^{A B} \mathcal{E}_{B}^{i}(H)\right)+ \\
& \left.+\partial_{i} \partial_{j}\left(\mathcal{E}_{A}^{i}(F) I^{A B} \mathcal{E}_{B}^{j}(H)\right)\right)
\end{aligned}
$$


Если $H$ - гамильтониан системы, то временная эволюция функционала $F$ задается дифференциальным уравнением $\dot{F}=\{F, H\}$. Данная скобка Пуассона обладает необходимыми свойствами антисимметричности, полноты и удовлетворяет тождествам Якоби [11]. Заметим, что уравнения движения с очевидностью следуют из вариационного принципа действия при учете поверхностных членов:

$$
\begin{aligned}
S & =\int_{V} d \mathbf{x}\left(\frac{1}{2} \dot{\varphi}^{A} I_{A B}^{-1} \varphi^{B}-h\left(\varphi^{A} ; \varphi_{, i}^{A}\right)\right) \\
\delta S & =\int_{V} d \mathbf{x}\left(\dot{\varphi}^{A} I_{A B}^{-1} \delta \varphi^{B}-\mathcal{E}_{B}(H) \delta \varphi^{B}-\partial_{i}\left(\mathcal{E}_{B}^{i}(H) \delta \varphi^{B}\right)\right) .
\end{aligned}
$$

Применим данный формализм к теории Янга-Миллса. Рассмотрим гамильтониан системы в конечной области $V$ :

$$
\mathcal{H}_{V}=\int_{V} d \mathbf{x}\left(\frac{1}{2}\left(E_{i}^{a}\right)^{2}+\frac{1}{4}\left(F_{i j}^{a}\right)^{2}-A_{0}^{a}\left(\partial_{i} E_{i}^{a}-g t^{a b c} A_{i}^{b} E_{i}^{c}\right)\right) .
$$

Переменная $E_{(1)}$ не является независимой. Поэтому любое граничное условие на нее будет дополнительной связью. Модифицируем гамильтониан $H_{V}=\mathcal{H}_{V}+\Delta H_{V}$ с помощью добавки поверхностного члена, содержащего эту связь с поверхностным множителем Лагранжа $A_{0}(R \widehat{\mathbf{x}})$,

$$
\Delta H_{V}=\int_{\partial V} d \breve{\mathbf{X}} A_{0}^{a}\left(\frac{h}{h_{1}} E_{(1)}^{a}-\chi^{a}\right),
$$

где $\chi=\chi(\breve{\mathbf{X}})$ - произвольная функция, задающая граничное условие, которую мы пока не конкретизируем. Отметим, что без добавки этого поверхностного члена уравнения движения в конечной области не локализуемы.

Предполагая обычную каноническую скобку Пуассона $I_{A_{i}^{a} E_{j}^{b}}=\delta^{a b} \delta_{i j}$, легко получить уравнения движения

$$
\begin{aligned}
\int_{V} d \mathbf{x} g_{i}^{a} \dot{A}_{i}^{a}= & \int_{V} d \mathbf{x} g_{i}^{a}\left(E_{i}^{a}+\nabla_{i} A_{0}^{a}\right) \\
\int_{V} d \mathbf{x} g_{i}^{a} \dot{E}_{i}^{a}= & \int_{V} d \mathbf{x} g_{i}^{a}\left(-g t^{a b c} E_{i}^{b} A_{0}^{c}+\nabla_{j} F_{i j}^{a}\right)- \\
& -\int_{\partial V} d \breve{\mathbf{X}} g_{(\alpha)}^{a} \frac{h}{h_{1}}\left(\frac{1}{h_{\alpha}} \partial_{(1)}\left(h_{\alpha} A_{(\alpha)}^{a}\right)-\frac{1}{h_{1}} \partial_{(\alpha)}\left(h_{1} A_{(1)}^{a}\right)\right) .
\end{aligned}
$$

При выводе поверхностного члена мы использовали следующие тождества на производные от базисных векторов:

$$
\begin{aligned}
& e_{j}^{(k)} \partial_{(k)} e_{j}^{(\alpha)}=\left(1-\delta^{k \alpha}\right) \frac{1}{h_{k} h_{\alpha}} \frac{\partial h_{k}}{\partial X_{\alpha}}, \\
& e_{j}^{(\beta)}\left(\partial_{(\alpha)} e_{j}^{(1)}-\partial_{(1)} e_{j}^{(\alpha)}\right)=\delta^{\alpha \beta} \frac{1}{h_{1} h_{\alpha}} \frac{\partial h_{\alpha}}{\partial X_{1}} .
\end{aligned}
$$

Интегралы от пробных функций пока нельзя удалить, т.е. временная эволюция определена только для распределений. Уравнение движения для переменной, канонически-сопряженной к $A_{0}$, дает гауссову связь

$$
\int_{V} d \mathbf{x} g^{a} \nabla_{i} E_{i}^{a}-\int_{\partial V} d \breve{\mathbf{X}} g^{a}\left(\frac{h}{h_{1}} E_{(1)}^{a}-\chi^{a}\right)=0 .
$$


Вследствие независимости значений пробных функций внутри области и на границе она распадается на два независимых условия:

$$
\nabla_{i} E_{i}^{a}=0, \quad \frac{h}{h_{1}} E_{(1)}^{a}(R, \breve{\mathbf{X}})=\chi^{a}(\breve{\mathbf{X}}) .
$$

Теперь зафиксируем обобщенную калибровку ФШ (3).

Заметим, что уравнение (17) фактически является локальным, поэтому с ним можно обращаться, как с уравнением для функции, а не как с распределением. Применим к обеим сторонам поперечной части уравнения операцию

$$
\frac{1}{h_{1} h_{\alpha}} \frac{\partial}{\partial X_{1}} h_{\alpha} .
$$

С помощью тождеств (20) и соотношения, которое является продольной частью (17), $\left(1 / h_{1}\right)\left(\partial A_{0}^{a} / \partial X_{1}\right)=-E_{(1)}^{a}$, получим следуюшее уравнение движения:

$$
\dot{F}_{(\alpha)(1)}^{a}=-g t^{a b c} F_{(\alpha)(1)}^{b} A_{0}^{c},
$$

где для более краткой записи мы использовали тождество

$$
\frac{1}{h_{\alpha}} \frac{\partial}{\partial X_{1}}\left(h_{\alpha} A_{(\alpha)}\right)=h_{1} F_{(\alpha)(1)} .
$$

Аналогично уравнение движения локально для продольной компоненты в (18), и мы получаем

$$
\dot{E}_{(1)}^{a}=-g t^{a b c} E_{(1)}^{b} A_{0}^{c}+G_{(1)}^{a} .
$$

С помошью несложных, но несколько громоздких выкладок, применяя (19), (20), мы можем доказать следующее тождество для продольной компоненты вектора $G_{i}=\nabla_{j} F_{i j}$ :

$$
G_{(1)}=-\frac{h_{1} h_{\alpha}}{h} \nabla_{(\alpha)}\left(\frac{h}{h_{1} h_{\alpha}} F_{(\alpha)(1)}\right) .
$$

Теперь мы готовы рассмотреть проблему выбора граничных условий. В соотношении (8), следуюшем из тождеств Бианки, а также соотношении, выражающем продольную компоненту электрического поля с помощью локальной гауссовой части (4), потребуем обычных условий регулярности полей в начале координат $\mathbf{x}^{0}$, т.е. выберем константу $X_{1}^{(0)}=x_{1}^{0}$ :

$$
\left.\frac{h}{h_{1}} B_{(1)}(\mathbf{X})\right|_{X_{1}=x_{1}^{0}}=0,\left.\quad \frac{h}{h_{1}} E_{(1)}(\mathbf{X})\right|_{X_{1}=x_{1}^{0}}=0 .
$$

В соотношении (6) на $G_{(1)}$, а также в нелокальной части связи выберем константу $\widetilde{X}_{1}^{(0)}=R$, что дает граничные условия на поверхности:

$$
\left.\frac{h}{h_{1}} G_{(1)}(\mathbf{X})\right|_{X_{1}=R}=0,\left.\quad \frac{h}{h_{1}} E_{(1)}(\mathbf{X})\right|_{X_{1}=R}=\chi(\breve{\mathbf{X}}),
$$

т.е. благодаря соотношению (26)

$$
F_{(\alpha)(1)}(R, \breve{\mathbf{X}})=0 .
$$


При таком граничном условии на $F_{(\alpha)(1)}$ уравнения движения для физических переменных (17), (18) становятся локальными. Множитель Лагранжа $A_{0}$ определяется легко:

$$
A_{0}(\mathbf{X})=A_{0}(R, \breve{\mathbf{X}})-\int_{R}^{X_{1}} d X_{1}^{\prime}\left(h_{1} E_{(1)}\right)\left(X_{1}^{\prime}, \breve{\mathbf{X}}\right)
$$

лишь с точностью до поверхностного множителя Лагранжа, который, вообще говоря, остается произвольным. Этот произвол отражает симметрию исходного действия относительно зависящих от времени калибровочных преобразований. Он фиксируется при задании граничных условий по времени, например периодических в статистической сумме при конечной температуре. Поэтому в температурной теории, вообше говоря, нельзя обратить поверхностный множитель Лагранжа в нуль выбором калибровочного преобразования.

Из уравнений (23), (25) видно, что все граничные условия, кроме поверхностной связи, сохраняются во времени и, таким образом, являются допустимыми. Однако из формулы $(25)$ видно, что величина $\chi(\breve{\mathbf{X}})$ не является константой, а изменяется во времени согласно закону

$$
\dot{\chi}^{a}(\breve{\mathbf{X}})=g t^{a b c} A_{0}^{b}(R, \breve{\mathbf{X}}) \chi^{c}(\breve{\mathbf{X}})
$$

Противоречие легко устраняется расширением фазового пространства системы. Рассмотрим $\chi$ как дополнительные гамильтоновы переменные такие, что их скобки Пуассона $\chi$ с обычными переменными равны нулю, а [13]

$$
\left\{\chi^{a}(\breve{\mathbf{X}}), \chi^{b}\left(\breve{\mathbf{X}}^{\prime}\right)\right\}=\omega^{a b}[\chi] \delta\left(\breve{\mathbf{X}}, \breve{\mathbf{X}}^{\prime}\right) \equiv g t^{a b c} \chi^{c}(\breve{\mathbf{X}}) \delta\left(\breve{\mathbf{X}}, \breve{\mathbf{X}}^{\prime}\right) .
$$

Тождество Якоби для таких скобок следует из тождества Якоби в алгебре Ли. Фазовым пространством $\Gamma_{\chi}$ данных переменных согласно симплектической конструкции Березина-Кириллова [13] являются орбиты коприсоединенного представления калибровочной группы. Для группы $S U(2)$ симплектическими пространствами $\Gamma_{\chi}$ будут сферы радиуса $|\chi|$ в коприсоединенном представлении. Уравнение (31) естественным образом совпадает с уравнением Гамильтона для данных переменных и выражается через действие группы в коприсоединенном представлении. Полное фазовое пространство системы есть прямое произведение $\widetilde{\Gamma}=\Gamma \times \Gamma_{\chi}$, где $\Gamma$ - фазовое пространство локализованных переменных.

Система обладает остаточной инвариантностью относительно группы $G_{\mathrm{res}}$ остаточных калибровочных преобразований. Генераторами этих преобразований являются поверхностные интегралы

$$
Q_{V}(g)=\int_{\partial V} d \breve{\mathbf{X}} g^{a}(\breve{\mathbf{X}})\left(\left(\frac{h}{h_{1}} E_{(1)}^{a}\right)(R, \breve{\mathbf{X}})-\chi(\breve{\mathbf{X}})\right)
$$

которые обладают алгеброй $\left\{Q_{V}(g), Q_{V}\left(g^{\prime}\right)\right\}=Q_{V}\left(-\left[g, g^{\prime}\right]\right)$, где $\left[g, g^{\prime}\right]^{a}=g t^{a b c} g^{b} g^{c}-$ цветовой коммутатор. Скобка Пуассона генераторов с гамильтонианом равна нулю, $\left\{Q_{V}(g), H_{V}\right\}=0$, а действие генераторов на калибровочное поле имеет вид $\left\{Q_{V}(g), A_{(\alpha)}(\mathbf{X})\right\}=\nabla_{(\alpha)} g(\breve{\mathbf{X}})$.

Таким образом, минимальное представление гамильтоновой динамики, инвариантное относительно преобразований симметрии и временной эволюции, оказывается прямым интегралом всех возможных представлений с различными направлениями $A_{0}$. Значения длин векторов $\chi$ и $A_{0}$ при этом фиксированы в каждом таком представлении и являются естественными параметрами порядка, нумеруюшими неприводимые представления динамики. 


\section{4. ЭФФЕКТИВНАЯ СТАТИСТИЧЕСКАЯ СУММА}

В данном разделе мы изучим зависимость статистической суммы абелевой и неабелевой калибровочных теорий от величины переменной на гранище $|\chi(\widehat{\mathbf{x}})|[14]$.

4.1. Электродинамика с внешним зарядом. Обозначим поперечные и продольные компоненты векторов по отношению к градиенту $\partial$ верхними индексами $\boldsymbol{\partial \mathbf { A } ^ { \perp }}=0$, а по отношению к радиус-вектору $\mathbf{x}-$ нижними $\mathbf{x} \mathbf{A}_{\perp}=0$. Будем работать в калибровке Кулона $\mathbf{A}=\mathbf{A}^{\perp}$. Заметим, что условие $\mathbf{A}=\mathbf{A}_{\perp}$ соответствует сферической калибровке ФШ [15]. Предположим также, что $V=\{\mathbf{x}:|\mathbf{x}|=R\}$ - сферическая область радиуса $R$. Тогда гауссова связь, $\boldsymbol{\partial E}=\rho(\mathbf{x})$, позволяет исключить одну из компонент электрического поля $\mathbf{E}$. Вьше мы показали, что граничные условия $\mathbf{E}_{\perp}(R \widehat{\mathbf{x}})=0$ и $x_{j} F_{i j}(R \widehat{\mathbf{x}})=0$ совместимы с временной эволюцией в калибровке $\Phi \amalg$. После перехода в калибровку Кулона мы получим соответствующее условие вида $\mathbf{E}^{\perp}(R \widehat{\mathbf{x}})=0$.

Статистическая сумма теории может быть представлена в виде следуюшего функционального интеграла:

$$
\begin{aligned}
Z= & \int \mathcal{D} \mathbf{A} \mathcal{D} \mathbf{E} \delta(\boldsymbol{\partial} \mathbf{A}) \delta\left(R^{2} E_{\|}(R \widehat{\mathbf{x}})-\chi(\widehat{\mathbf{x}})\right) \times \\
& \times \exp \int_{\Lambda} d^{4} x\left(i \mathbf{E} \dot{\mathbf{A}}-\frac{1}{2} \mathbf{E}^{2}+\frac{1}{2} \mathbf{A} \Delta \mathbf{A}-\frac{1}{2 \epsilon^{2}}(\boldsymbol{\partial} \mathbf{E}-\rho)^{2}\right),
\end{aligned}
$$

где было введено обозначение $\Lambda=[0, \beta] \times V$ (мы также будем пользоваться записью $\partial \Lambda=[0, \beta] \times \partial V)$. Гауссова связь регуляризована с помощью малого параметра $\epsilon$, который нужно положить равным нулю в конще вычислений. Данньй гауссов интеграл вычисляется стандартным образом с помощью сдвига переменных интегрирования. Для выгисления интеграла по $\mathbf{E}$ введем новую переменную интегрирования $\mathbf{E}_{1}$ :

$$
\mathbf{E}=\mathbf{E}_{1}+\mathcal{E}, \quad \mathbf{E}_{1}(R \widehat{\mathbf{x}})=0 .
$$

Здесь новая переменная $\mathbf{E}_{1}$ удовлетворяет нулевому граничному условию и $\mathcal{E}$ выбрано так, чтобы отсутствовал линейный член по $\mathbf{E}_{1}$. Это дает следующее уравнение для определения $\mathcal{E}$ :

$$
\begin{aligned}
& i \dot{\mathbf{A}}-\mathcal{E}+\frac{1}{\epsilon^{2}} \boldsymbol{\partial}(\boldsymbol{\partial \mathcal { E }})-\frac{1}{\epsilon^{2}} \partial \rho=0, \\
& R^{2} \widehat{\mathbf{x}} \mathcal{E}(R \widehat{\mathbf{x}})=\chi(\widehat{\mathbf{x}}) .
\end{aligned}
$$

Последнее граничное условие следует из второй дельта-функции в (34). Можно разложить данный вектор на продольную и поперечную компоненты в импульсном пространстве $\mathcal{E}=\mathcal{E}^{\perp}-\boldsymbol{\partial} \varphi$. Тогда поперечная часть равна $\mathcal{E}^{\perp}=i \dot{\mathbf{A}}$ и уравнение для $\varphi$ принимает Вид

$$
\left(\Delta-\epsilon^{2}\right) \varphi=-\rho, \quad R^{2} \frac{\partial \varphi}{\partial R}=-\chi(\widehat{\mathbf{x}}) .
$$

Статистическая сумма (34) может быть представлена в виде произведения

$$
\begin{aligned}
Z & =Z_{1} \widetilde{Z} \\
Z_{1} & =\int \mathcal{D} \mathbf{A}^{\perp} \mathcal{D} \mathbf{E} \exp \int_{\Lambda} d^{4} x\left(-\frac{1}{2} \dot{\mathbf{A}}^{2}+\frac{1}{2} \mathbf{A} \Delta \mathbf{A}-\frac{1}{2} \mathbf{E}_{1}^{2}-\frac{1}{2 \epsilon^{2}}\left(\boldsymbol{\partial \mathbf { E } _ { 1 } ) ^ { 2 }}\right),\right. \\
\widetilde{Z} & =\exp \beta\left(\frac{1}{2} \int_{\partial V} d \widehat{\mathbf{x}} \chi(\widehat{\mathbf{x}}) \varphi(R \widehat{\mathbf{x}})-\frac{1}{2} \int_{\partial V} d \mathbf{x} \rho(\mathbf{x}) \varphi(\mathbf{x})\right)
\end{aligned}
$$


где $\varphi$ - это решение (38).

Решение (38), очевидно, является суммой однородной части $\phi$, удовлетворяюшей нетривиальному граничному условию, и неоднородной части, удовлетворяющей нулевому граничному условию:

$$
\varphi=\phi-G \bullet \rho, \quad G=\left(\Delta-\epsilon^{2}\right)^{-1},
$$

где $G$ - функция Грина с нулевым граничным условием Неймана, а • обозначает интегрирование по области $\Lambda$. Введем следующие обозначения:

$$
\begin{aligned}
\widetilde{Z} & =\widetilde{Z}_{\chi} \widetilde{Z}_{\rho \rho} \widetilde{Z}_{\rho \chi}, \\
\widetilde{Z}_{\chi} & =\exp \left(\frac{\beta}{2} \int_{\partial V} d \widehat{\mathbf{x}} \chi(\widehat{\mathbf{x}}) \phi(\widehat{\mathbf{x}})\right), \\
\widetilde{Z}_{\rho \rho} & =\exp \left(\frac{\beta}{2} \int_{\partial V} d \mathbf{x} d \mathbf{y} \rho(\mathbf{x}) G(\mathbf{x}, \mathbf{y}) \rho(\mathbf{y})\right), \\
\widetilde{Z}_{\rho \chi} & =\exp \left(-\frac{\beta}{2} \int_{\partial V} d \mathbf{x} \phi(\mathbf{x}) \rho(\mathbf{x})\right) \exp \left(-\frac{\beta}{2} \int_{\partial V} d \widehat{\mathbf{x}} \chi(\widehat{\mathbf{x}})(G \bullet \rho)(\widehat{\mathbf{x}})\right) .
\end{aligned}
$$

Естественно рассмотреть данную проблему в терминах сферических координат. Решение (38), регулярное внутри сферы, дается формулой

$$
\phi_{l m}=C_{l m} \sqrt{\frac{\pi}{2 \epsilon r}} I_{l+1 / 2}(\epsilon r) .
$$

Постоянная $C_{l m}$ при этом определяется уравнением (38). Роль регуляризатора $\epsilon$ теперь становится понятной. Решением для нулевой моды будет

$$
\phi_{00}=C_{00} \frac{\operatorname{sh} \epsilon r}{\epsilon r}, \quad C_{00}=-\frac{\chi_{00}}{R^{2} \epsilon\left(\operatorname{ch} \frac{\epsilon R}{\epsilon R}-\operatorname{sh} \frac{\epsilon R}{(\epsilon R)^{2}}\right)},
$$

оно имеет $1 / \epsilon^{2}$-сингулярность при стремлении $\epsilon$ к нулю. В то же время решения для других мод регулярны в этом пределе и стремятся к

$$
\phi_{l m}=C_{l m} r^{l}, \quad C_{l m}=-\frac{\chi_{l m}}{l R^{l+1}} .
$$

В рассмотрении нулевой моды необходимо соблюдать аккуратность и удерживать конечное $\epsilon$. Функция Грина для нулевой моды определяется как решение уравнений

$$
\begin{aligned}
& \left(\frac{1}{r} \frac{\partial^{2}}{\partial r^{2}} r-\epsilon^{2}\right) G_{00}\left(r, r^{\prime}\right)=\frac{\delta\left(r-r^{\prime}\right)}{r r^{\prime}}, \\
& \left.\frac{\partial G_{00}\left(r, r^{\prime}\right)}{\partial r}\right|_{r=R}=0
\end{aligned}
$$

и легко может быть вычислена в явном виде

$$
G_{00}\left(r, r^{\prime}\right)=\frac{1}{\epsilon r r^{\prime}}\left(\frac{1}{2} \operatorname{sh} \epsilon\left(r-r^{\prime}\right)-\frac{1}{2} \operatorname{sh} \epsilon\left(r+r^{\prime}\right)+\frac{\operatorname{sh} \epsilon R-\operatorname{ch} \frac{\epsilon R}{\epsilon R}}{\operatorname{ch} \epsilon R-\operatorname{sh} \frac{\epsilon R}{\epsilon R}} \operatorname{sh} \operatorname{sh} \epsilon r^{\prime}\right) .
$$


Ведущие члены в разложении Лорана при малом $\epsilon$ имеют вид

$$
\begin{aligned}
& G_{00}\left(r, r^{\prime}\right) \simeq-\frac{3}{\epsilon^{2} R^{3}}+\frac{9}{5 R}-\frac{1}{2} \frac{r^{2}+r^{\prime 2}}{R^{3}}-\frac{1}{\max \left(r, r^{\prime}\right)} \\
& \phi_{00} \simeq-\left(\frac{3}{\epsilon^{2} R^{3}}-\frac{3}{10 R}+\frac{r^{2}}{2 R^{3}}\right) \chi_{00}
\end{aligned}
$$

Подстановка этих выражений в формулы (41) дает для ненулевых мод в пределе $\epsilon=0$ следующее выражение:

$$
\widetilde{Z}_{\chi l>0}=\exp \left(-\frac{\beta}{2 R} \sum_{l m>0} \frac{\left|\chi_{l m}\right|^{2}}{l}\right) .
$$

Для простоты будем предполагать, что распределение заряда $\rho$ является сферическисимметричным. В этом случае оно дает вклад только в нулевую моду. Введем обозначения для интегралов:

$$
\mathcal{Q}_{00}=\int_{0}^{R} r^{2} d r \rho_{00}(r), \quad \mathcal{G}_{00}=\int_{0}^{R} r^{4} d r \rho_{00}(r)
$$

Тогда результат можно представить в виде

$$
\begin{aligned}
\widetilde{Z}_{00}= & \exp \beta\left(-\frac{1}{2} \int_{0}^{R} x^{2} d x y^{2} d y \frac{\rho_{00}(x) \rho_{00}(y)}{\max (x, y)}-\frac{3}{2 \epsilon^{2} R^{3}}\left(\mathcal{Q}_{00}-\chi_{00}\right)^{2}+\right. \\
& \left.+\frac{1}{R}\left(\frac{9}{10} \mathcal{Q}_{00}^{2}-\frac{1}{10} \chi_{00}^{2}-\frac{3}{10} \chi_{00} \mathcal{Q}_{00}\right)-\frac{1}{2 R^{3}}\left(\mathcal{Q}_{00}-\chi_{00}\right) \mathcal{G}_{00}\right)
\end{aligned}
$$

В пределе $\epsilon \rightarrow 0$ это выражение будет содержать дельта-функцию от условия $\mathcal{Q}_{00}=$ $\chi_{00}$, и мы приходим к следуюшей поправке к стандартному ответу в результате вклада поверхностных членов:

$$
\widetilde{Z}_{00}=\exp \left(\frac{\beta}{2 R} \mathcal{Q}_{00}^{2}\right) \delta\left(\mathcal{Q}_{00}-\chi_{00}\right)
$$

В качестве простой иллюстрации полученного результата рассмотрим плотность заряда $\rho_{00}=-\kappa /(\sqrt{\pi} r)$, соответствуюшую линейно растушему потенциалу $\varphi=\kappa r$. Для такого экзотического распределения заряда, моделируюшего конфайнментный потеншиал, мы получим дополнительный константный вклад в плотность свободной энергии

$$
\Delta \mathcal{F}=-\frac{\ln Z}{\beta V}=-\frac{3}{32 \pi^{2}} \kappa^{2}
$$

Интересно подчеркнуть, что своим возникновением он полностью обязан граничным членам и эта добавка уменьшает свободную энергию. Данный пример указывает на возможную связь между явлением конфайнмента в неабелевой калибровочной теории и граничными эффектами. 
4.2. $S U(2)$-глюодинамика. В предыдущей работе [16] было выведено представление для статистической суммы глюодинамики $Z$ в виде функционального интеграла по коллективным переменным. Для того чтобы обобшить это представление на эффективную статистическую сумму, вернемся к исходному моменту вычислений в калибровке $\Phi \amalg$ :

$$
\begin{aligned}
Z[\chi]= & \int \mathcal{D} \mathbf{A} \mathcal{D} \mathbf{E} \mathcal{D} \sigma \exp \left(\int _ { \Lambda } d ^ { 4 } x \left(i \mathbf{E} \dot{\mathbf{A}}-\frac{1}{2} \mathbf{E}^{2}-\frac{1}{2} \mathbf{B}^{2}+\right.\right. \\
& +i \sigma \nabla \mathbf{E})) \delta\left(A_{\|}\right) \delta\left(R^{2} E_{\|}(R \widehat{\mathbf{x}})-\chi\right),
\end{aligned}
$$

где $\sigma$, по существу, является другим обозначением временной компоненты калибровочного поля $A_{0}$. Легко видеть, что зависимость от переменной $\chi$ полностью сконцентрирована в интеграле по $E_{\|}$:

$$
I=\int \mathcal{D} E_{\|} \exp \left(\int_{\Lambda} d^{4} x\left(-\frac{1}{2} E_{\|}^{2}+i \sigma(\boldsymbol{\partial} \widehat{\mathbf{x}}) E_{\|}\right)\right) \delta\left(R^{2} E_{\|}-\chi\right) .
$$

Последний интеграл вычисляется с помощью сдвига $E_{\|}=E_{\|}^{1}+\mathcal{E}$, где $\mathcal{E}=-i \partial \sigma / \partial x$ и $E_{\|}^{1}$ удовлетворяет нулевому граничному условию Дирихле, что дает

$$
I=\exp \left(\int_{0}^{\beta} d t\left[-\frac{1}{2} \int_{V} d \mathbf{x}\left(\frac{\partial \sigma}{\partial x}\right)^{2}+i \int d \widehat{\mathbf{x}} \sigma \chi\right]\right) \delta\left(R^{2} \frac{\partial \sigma}{\partial R}-i \chi\right) .
$$

Далее, вводя представление в виде следующего функционального интеграла:

$$
\exp \left(-\frac{1}{2} \int_{\Lambda} d^{4} x B_{\|}^{2}\right)=\int \mathcal{D} \nu \exp \left(\int_{\Lambda} d^{4} x\left(-\frac{1}{2} \nu^{2}+i \nu B_{\|}\right)\right)
$$

после интегрирования по $\mathbf{A}_{\perp}$ и $\mathbf{E}_{\perp}$ в конечном итоге приходим к результату (см. подробнее в [16])

$$
\begin{aligned}
& Z[\chi]=\int \mathcal{D} \sigma \mathcal{D} \nu \exp \left(-W[\sigma, \nu]+i \int_{\partial \Lambda} d t d \widehat{\mathbf{x}} \sigma \chi\right) \delta\left(R^{2} \sigma^{\prime}-i \chi\right), \\
& 2 W[\sigma, \nu]=\nu \bullet \nu+\boldsymbol{\partial} \sigma \bullet \boldsymbol{\partial} \sigma+\mathbf{K}_{-} \bullet C_{+}^{-1} \bullet \mathbf{K}_{+}+ \\
& +\mathbf{K}_{+} \bullet C_{-}^{-1} \bullet \mathbf{K}_{-}+\operatorname{tr} \ln C_{+} C_{-}, \\
& C_{ \pm}=-\Delta_{x}-\nabla_{t}^{2} \pm D, \quad \mathbf{K}_{ \pm}=\boldsymbol{\partial}_{ \pm} \nu \pm \nabla_{t} \boldsymbol{\partial}_{ \pm} \sigma, \\
& \nabla_{t}^{a b}=\delta^{a b} \partial_{t}-g t^{a b c} \sigma^{c}, \quad D^{a b}=g t^{a b c} \nu^{c},
\end{aligned}
$$

где спроецированные производные определены как

$$
\partial_{ \pm}^{i}=\Pi_{ \pm}^{i j} \partial_{j}, \quad \Pi_{ \pm}^{i j}=\frac{1}{2}\left(\delta^{i j}-\hat{x}^{i} \hat{x}^{j} \pm i \epsilon^{i j k} \hat{x}^{k}\right) .
$$

В приближении стационарной фазы необходимо разложить эффективное действие в окрестности седловой точки:

$$
W\left[\varsigma+\sigma_{1}\right]=W[\varsigma]+\int_{\Lambda} d^{4} x \frac{\delta W}{\delta \varsigma(\mathbf{x})} \sigma_{1}(\mathbf{x})+\int_{\partial \Lambda} d t R^{2} d \widehat{\mathbf{x}} \mathcal{E}^{(1)}[\varsigma] \sigma_{1}(R \widehat{\mathbf{x}})+\cdots
$$


Нулевое приближение среднего поля дает

$$
\begin{aligned}
& \widetilde{Z}[\chi]=\exp \left(-W[\varsigma]+i \int_{\partial \Lambda} d t d \widehat{\mathbf{x}} \varsigma \chi\right), \\
& \frac{\delta W}{\delta \varsigma}=0, \quad R^{2} \frac{\partial \varsigma(R \widehat{\mathbf{x}})}{\partial R}=i \chi
\end{aligned}
$$

где вклад первой производной Эйлера $\mathcal{E}^{(1)}[\varsigma]$ точно сокрашается с поверхностным членом в (59). Таким образом, в приближении среднего поля зависимость $Z[\chi]$ полностью определяется решениями ऽ приближения стационарной фазы. Как мы показали вьше, абелева теория допускает только тривиальное решение $\varsigma=0$.

В работе [16] изучены постоянные решения приближения стационарной фазы для неабелевой теории. Кратко воспроизведем эти результаты здесь, в дополнение удерживая конечньй объем. Прежде всего введем обозначения для плотности свободной энергии $\mathcal{F}_{R}$ :

$$
W_{R}=\beta V_{R} \mathcal{F}_{R}, \quad \mathcal{F}_{R}=\gamma_{R} F_{R}, \quad \gamma_{R}=\frac{8 \pi^{2} R \delta(\hat{0})}{\beta^{2} V_{R}}
$$

где $V_{R}=4 \pi R^{3} / 3$ - объем шара и

$$
\delta(\hat{0})=\frac{1}{4 \pi} \sum_{l}(2 l+1)
$$

- ультрафиолетово расходящаяся угловая дельта-функция при совпадающих аргументах. Функцию $F_{R}$ можно выразить через безразмерные переменные:

$$
\sigma=\frac{2 \pi s}{\beta g}, \quad \nu=i\left(\frac{2 \pi u}{\beta g}\right)^{2}
$$

где, для того чтобы иметь действительное значение среднего магнитного поля, $\nu$ должно быть чисто мнимым (см. (58)). Вводя контрольный параметр $a=(2 \pi)^{4} /\left(2 g^{2} \beta^{4} \gamma_{R}\right)$ и осушествляя некоторые вычисления, мы получим

$$
\begin{aligned}
& F_{R}[u, s]=-a u^{4}+\mathcal{U}_{R}[u, s] \\
& \mathcal{U}_{R}[u, s]=\mathcal{U}_{R}[s]+\mathcal{V}_{R}[u, s] \\
& \mathcal{V}_{R}[u, s]=\frac{\beta}{2 \pi R} \sum_{n=-\infty}^{\infty} \ln \frac{L_{R}\left((n+s)^{2}+u^{2}\right) L_{R}\left((n+s)^{2}-u^{2}\right)}{L_{R}^{2}\left((n+s)^{2}\right)} \\
& \mathcal{U}_{R}[s]=\frac{\beta}{\pi R} \sum_{m=0}^{\infty} \ln \left(1-\frac{\cos 2 \pi s}{\operatorname{ch}\left(\pi\left(m+\frac{1}{2}\right) \frac{\beta}{R}\right)}\right)
\end{aligned}
$$

где $L_{R}(x)=\operatorname{ch}(2 \pi R \sqrt{x} / \beta)$.

Можно показать, что при конечном радиусе $R$ эта свободная энергия обладает только тривиальным минимумом при $s=u=0$. Ситуация меняется после взятия термодинамического предела $R \rightarrow \infty$. Результируюшее выражение для свободной энергии (см. [16]) при высокой температуре также обладает только тривиальным минимумом 
$u=s=0$. Однако при некоторой критической температуре происходит фазовый переход первого рода, так что ниже этой температуры существует более глубокий нетривиальный минимум при $u=s=1 / 2$ (см. [16]).

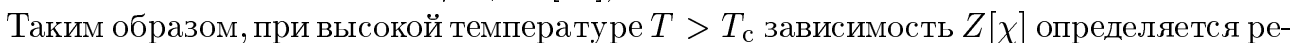
шением линеаризованного уравнения $\delta^{2} W / \delta \varsigma^{2} \bullet \sigma_{1}$ в окрестности $\varsigma=0$. Это приводит лишь к зависимости аналогичной абелевой теории. А именно, эффективная статистическая сумма содержит дельта-функцию, выражаюшую закон сохранения глобального заряда, но в остальном тривиальна в том смысле, что $Z\left[\chi_{l m}\right] \rightarrow 1$ в термодинамическом пределе $R \rightarrow \infty$. Эта ситуация, очевидно, соответствует фазе деконфайнмента, в которой нет никаких ограничений на потоки цветового заряда на бесконечности.

Однако при температуре ниже критической $T<T_{\mathrm{c}}$ сушествует постоянное ненулевое решение $|\varsigma|=\pi / g \beta$. Поскольку система инвариантна относительно группы больших калибровочных преобразований $G_{\infty}$, параметризуемой матрицами $U(\widehat{\mathbf{x}})$, соответствующий единичный цветовой вектор $\hat{\boldsymbol{s}}(\widehat{\mathbf{x}})$ произволен в каждом направлении $\widehat{\mathbf{x}}$. После интегрирования по орбитам группы $S U(2)$ в каждом конусе $\widehat{\mathbf{x}}$ получаем

$$
\widetilde{Z}[\chi]=\prod_{\widehat{\mathbf{x}}} \frac{\sin \Delta \beta \varsigma \chi(\widehat{\mathbf{x}})}{\Delta \beta \varsigma \chi(\widehat{\mathbf{x}})} \sim \exp \left(-\frac{\Delta \pi^{2}}{g^{2}} \int_{\partial V} d \widehat{\mathbf{x}} \chi^{2}(\widehat{\mathbf{x}})\right),
$$

где введена дискретизация единичной сферы, а $\Delta$ - инфинитезимальный элемент плошади. Хорошо известно [3], что в непрерывном пределе "голая" константа связи исчезает $g \rightarrow 0$, тем самым делая $Z[\chi]$ функцией с очень резким максимумом вокруг нулевого аргумента. Это соответствует фазе конфайнмента, в которой цветовые потоки на бесконечности равны нулю в каждом угловом направлении.

Таким образом, эффективная статистическая сумма зависит от $\chi$ следующим обра3ом:

$$
Z[\chi]= \begin{cases}\prod_{\widehat{\mathbf{x}}} \delta(\chi(\widehat{\mathbf{x}})), & T<T_{\mathrm{c}} \\ 1, & T>T_{\mathrm{c}} .\end{cases}
$$

Следовательно, температурное среднее некоторой наблюдаемой $A$ дается формулой

$$
\langle A\rangle= \begin{cases}\langle A\rangle_{0}, & T<T_{\mathrm{c}} \\ \int d \chi(\widehat{\mathbf{x}})\langle A\rangle_{\chi}, & T>T_{\mathrm{c}}\end{cases}
$$

где введены средние по неэквивалентным “секторам":

$$
\langle A\rangle_{\chi}=\frac{1}{Z[\chi]} \int \mathcal{D} \sigma \mathcal{D} \nu \exp \left\{-W[\sigma, \nu]+i \int_{\partial \Lambda} d t d \widehat{\mathbf{x}} \sigma \chi\right\} A[\sigma, \nu] .
$$

Легко видеть, что при низкой температуре гиббсовские средние содержат синглетный проектор $P_{s}$ группы больших калибровочных преобразований $G_{\infty}$,

$$
\langle A\rangle_{0}=\lim _{R \rightarrow \infty} \frac{1}{Z_{R}[0]} \operatorname{Tr}\left(e^{-\beta H_{R}} \delta\left(Q_{R}\right) A\right)=\lim _{R \rightarrow \infty} \frac{1}{Z_{R}[0]} \operatorname{Tr}\left(e^{-\beta H_{R}} P_{s} A\right),
$$

где

$$
Q_{R}=\int_{V_{R}} d \mathbf{x} \nabla \mathbf{E}
$$

- оператор цветового заряда в объеме $V$. Наличие такого проектора в средних ведет, в свою очередь, к закону плошадей для вильсоновской петли $[16,17]$, что рассматривается как стандартньй критерий конфайнмента. 


\section{5. ЗАКЛЮЧЕНИЕ}

Гауссова связь калибровочной теории может быть точно разрешена в физической калибровке. Это приводит к эффективно нелокальным взаимодействиям, порождаюшим граничную нетривиальность теории.

Мы изучили зависимость эффективной статистической суммы от граничного условия Дирихле $R^{2} E_{\|}(R \widehat{\mathbf{x}})=\chi(\widehat{\mathbf{x}})$, налагаемого на вспомогательную продольную компоненту электрического поля в электродинамике с внешним зарядом и $S U(2)$-глюодинамике. В абелевом случае такая зависимость всегда содержит дельта-функцию, выражаюшую закон сохранения глобального заряда, а также нетривиальную поправку к плотности свободной энергии для распределений плотности заряда, медленно убывающих на пространственной бесконечности.

Неабелев характер взаимодействия приводит к более необычному эффекту. При низких температурах ограничение возможных граничных значений продольной компоненты электрического поля проявляется в механизме конфайнмента, предложенного ранее в работе [16]. В самом деле, такая величина пропорциональна потоку цветового заряда в инфинитезимальном конусе $\widehat{\mathbf{x}}$ на пространственной бесконечности. Таким образом, поскольку цветовые потоки равны нулю в каждом направлении, цветной заряд локализован и не может экспериментально наблюдаться вне конечной областивзаимодействия.

Э.Г. Тимошенко хотел бы горячо поблагодарить проф. Б.А. Арбузова, проф. В. И. Саврина, а также д.ф.-м.н. Э.Э. Бооса и В.А. Ильина, к.ф.-м.н. В. О. Соловьева и Ю.А. Кузнецова за многочисленные полезные обсуждения и ценные замечания.

\section{Список литературы}

[1] S. Mandelstam. Phys. Rep. 1980. V. 67. P. 109.

[2] M. Bander. Phys. Rep. 1981. V. 75 (4). P. 205.

[3] L.D. Faddeev, A.A. Slavnov. Gauge Fields. Introduction to Quantum theory. N. Y.: Benjamin, 1980 .

[4] B. Е. Захаров. ПМТФ. 1968. Т. 3. С. 86.

[5] V.S. Buslaev, L. D. Faddeev, L. A. Takhtajan. Physica D. 1986. V. 18. P. 255.

[6] D. Lewis, J. Marsden, R. Montgomery, T. Ratiu. Physica D. 1986. V. 18. P. 391.

[7] T. Regge, C. Teitelboim. Ann. Phys. (N.Y.). 1974. V. 88. P. 286.

[8] V. O. Soloviev. Phys. Lett. B. 1992. V. 292. P. 30.

[9] A. Balachandran, G. Bimonte, K.S. Gupta, A. Stern. Int. J. Mod. Phys. A. 1992. V. 7. P. 4655.

[10] A. Balachandran, L. Chandar, E. Ercolessi et al. Int. J. Mod. Phys. A. 1994. V. 9. P. 3417; A. Balachandran, G. Bimonte, K.S. Gupta, A. Stern. Int. J. Mod. Phys. A. 1992. V. 7. P. 4655.

[11] V. O. Soloviev. J. Math. Phys. 1993. V. 34. P. 5747.

[12] G. Morchio, F. Strocchi. J. Math. Phys. 1987. V. 28 (3). P. 622; Commun. Math. Phys. 1985. V. 99. P. 153; J. Math. Phys. 1987. V. 28 (8). P. 1912.

[13] A. А. Кириллов. Элементы теории представлений. М.: Наука, 1972.

[14] N. A. Sveshnikov, E. G. Timoshenko. Phys. Rev. D. 1998 (in print).

[15] B. А. Фок. ЖЭТФ. 1937. Т. 12 (4). C. 404; J. Schwinger. Phys. Rev. 1951. V. 82. P. 664; D. Shütte. Phys. Rev. D. 1989. V. 40. P. 2090; N. A. Sveshnikov, E. G. Timoshenko. Confinement phase transition mechanism of $S U(2)$-gluodynamics. Preprint IHEP 91-140. Serpukhov: IHEP, 1991.

[16] N. A. Sveshnikov, E. G. Timoshenko. Phys. Lett. B. 1992. V. 289. P. 423.

[17] Э.Г. Тимошенко. ЯФ. 1993. Т. 56 (11). С. 277.

Поступила в редакцию 14.IV.1998 г. 